\title{
Familial aggregation of streptomycin ototoxicity: autosomal dominant inheritance?
}

\author{
D L VILJOEN*, S L SELLARS $\dagger$, AND P BEIGHTON $\ddagger$ \\ From the Departments of Human Genetics* $\ddagger$ and Otorhinolaryngology $\dagger$, Medical School and \\ Groote Schuur Hospital, University of Cape Town, Cape Town, South Africa.
}

SUMMARY Eight members of a large kindred of mixed ancestry from a remote rural area of South Africa were investigated for deafness. In each, severe permanent perceptive hearing loss had developed during antituberculous therapy with streptomycin sulphate in conventional doses. Although unproven by the data available in this study, the familial aggregation and pattern of distribution of sensitivity to streptomycin suggested autosomal dominant inheritance.

Streptomycin sulphate is frequently administered routinely as a primary drug in the treatment of pulmonary tuberculosis. The serious ototoxic effects of this drug which occasionally develop are related to the dosage, blood concentration, age of the patient, and renal function. Clustering of untoward reactions within families treated with conventional doses of streptomycin has been noted and in some instances this predisposition has apparently been inherited. ${ }^{1-3}$

We have investigated a large kindred in which eight persons developed severe perceptive deafness during antituberculous therapy with parenterally administered streptomycin sulphate. Our findings are presented and discussed in this paper in order to support the concept of a rare autosomal gene which confers a high risk for a dominant phenotype of permanent perceptive deafness upon exposure to conventional treatment with streptomycin.

\section{Investigation, subjects, and methods}

A large non-consanguineous family of mixed ancestry in which several persons had deafness that was related to antituberculous therapy was seen in a peripheral genetic clinic. The kindred consisted of persons of social class $\mathrm{V}$ and numerous members had suffered from pulmonary tuberculosis.

The deaf persons in the family, eight in all, were admitted to Groote Schuur Hospital, Cape Town, for appraisal and investigation. A previous history of ear

Received for publication 7 December 1982. Accepted for publication 22 February 1983. trauma or infection, renal dysfunction, and specific diseases such as mumps and meningoencephalitis was sought. Specific enquiry was made concerning the time of onset of deafness, tinnitus, and vertigo in relation to tuberculosis treatment. Details of drugs, dosages, and duration of administration were obtained from records at the community treatment clinic.

Routine clinical examination was performed and any concurrent medical conditions were treated. The following special tests of hearing and vestibular function were undertaken: pure tone and speech discrimination tests; tympanometry; the Carhart or Jerger tone decay test; Luschers recruitment test; Bekesy audiometry; electronystagmographic assessments of spontaneous and positional nystagmus; pendulum tracking tests; and the Hallpike bithermal (caloric) induced nystagmus tests.

Several other members of the kindred had retained normal hearing despite antituberculous therapy. For logistical reasons, these persons could not all be brought to Cape Town for investigation. However, every endeavour was made to secure information concerning key subjects.

\section{Results}

The pedigree of the kindred is illustrated in the figure. This shows four generations in which eight persons became deaf while on antituberculous therapy. Five additional family members received antituberculous treatment with no apparent effect on hearing, although only one of these (III.50) was given 


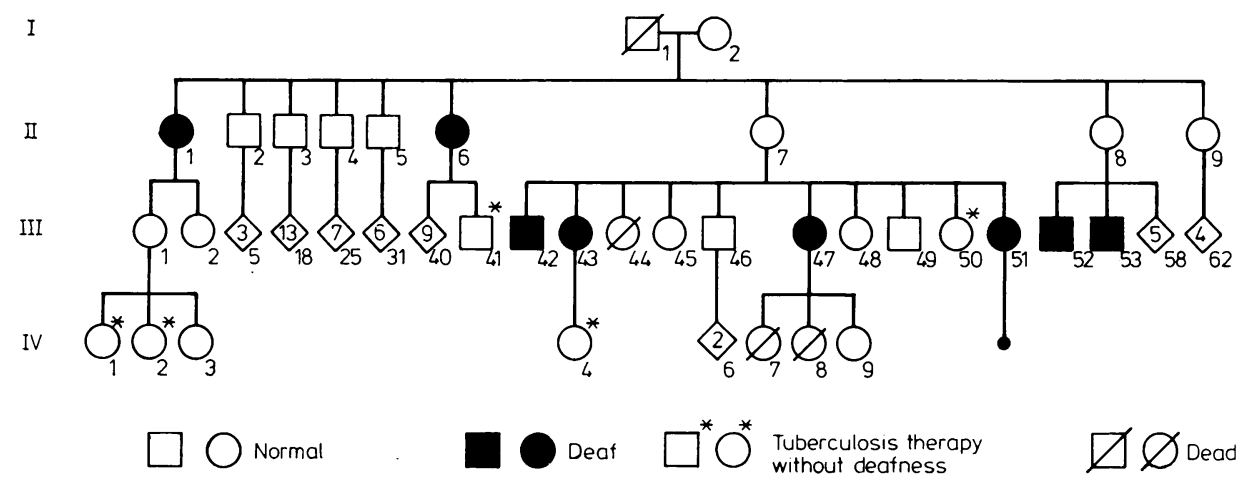

FIGURE Pedigree of affected family.

streptomycin. Both sexes are involved and the pattern of transmission is compatible with, but does not prove, autosomal dominant inheritance.

Basic information concerning the deaf persons is listed in table 1. This includes age at start of treatment, sex, drugs administered together with dosages, duration of therapy, and total dosage, and time and mode of onset of symptoms. It is noteworthy that six of the eight patients were adolescents or young adults when they were treated, while one patient was 6 years of age and the other 49 years of age.

The dose of streptomycin sulphate received by affected persons ranged from 13.0 to $20.0 \mathrm{mg} / \mathrm{kg}$ body weight daily. Therapy details are incomplete for three patients but, as far as could be ascertained, none received an amount in excess of $20 \mathrm{mg} / \mathrm{kg}$ body weight per day. (Dose related ototoxic side effects are known to increase markedly above this level.) Total dosages of streptomycin administered averaged $75.5 \mathrm{~g}$ per patient in the five subjects for whom accurate records were available.

Those patients with hearing loss (table 2) had $\frac{\mathbb{\Phi}}{-}$ bilateral moderately severe to very severe sensori- $\vec{\omega}$ neural deafness, although in four persons the involve- $\stackrel{\infty}{\omega}$ ment differed in degree between the ears. The speech audiometry results were poor in all these patients, with only five ears attaining word discrimination scores of over $60 \%$ at optimal intensity.

The tympanograms were normal, as would be $\frac{\mathscr{Q}}{\mathscr{D}}$ expected from the normal clinical and otoscopical findings in all patients, except in III.43 (patient 4) $\overrightarrow{\overrightarrow{0}}$ who had a large perforation of the left tympanic

TABLE 1 Basic data concerning the deaf patients.

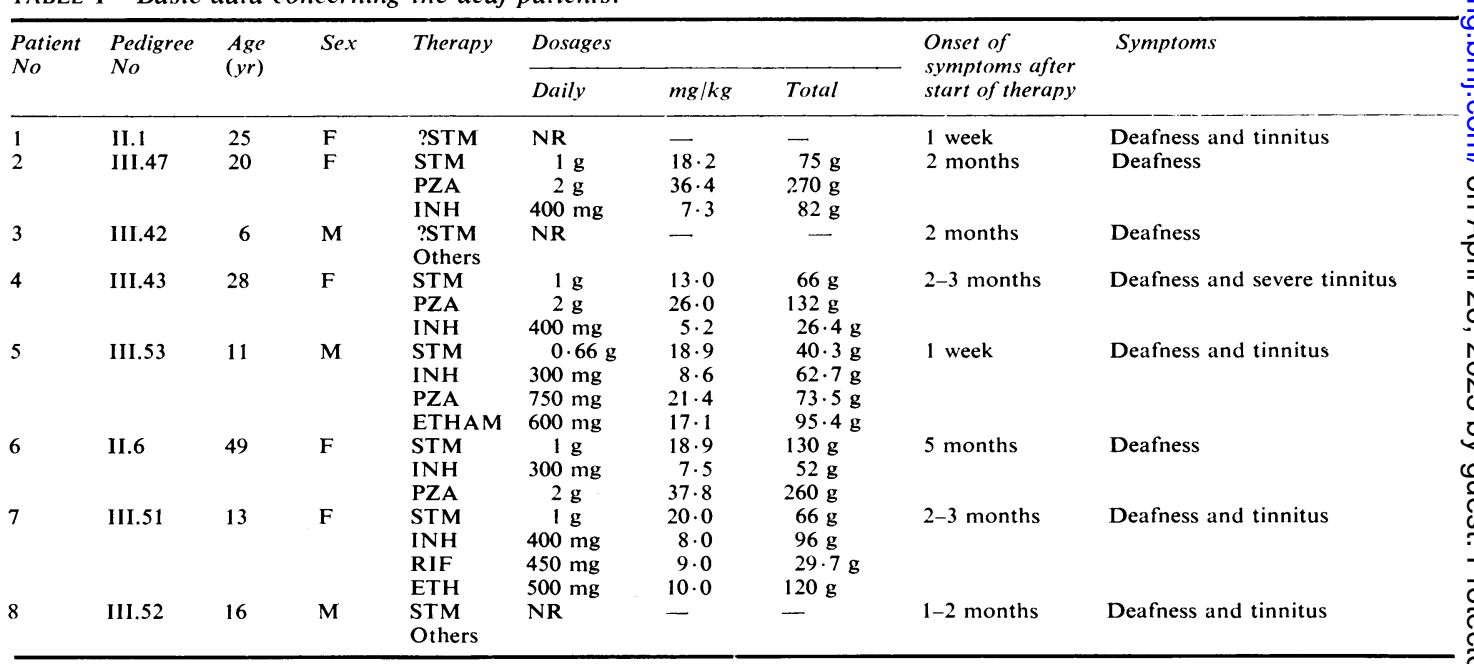

STM $=$ streptomycin sulphate, $\mathbf{I N H}=$ isoniazid, $\mathbf{P Z A}=$ pyrazinamide, $\mathrm{RIF} \approx$ rifampicin, $\mathbf{E T H A M}=$ ethambutal, $\mathrm{ETH}=$ ethionamide, NR $=$ not recorded. 
TABLE 2 Audiometric and vestibular function studies.

\begin{tabular}{|c|c|c|c|c|c|}
\hline \multirow[t]{2}{*}{ Patient } & \multicolumn{2}{|l|}{ Pure tone test } & \multicolumn{2}{|c|}{ Speech discrimination score $(\%)$} & \multirow[t]{2}{*}{ ENG findings (calorics) } \\
\hline & Right & Left & Right & Left & \\
\hline 1 & Moderately severe & Severe & 60 & 60 & Normal \\
\hline 2 & Moderately severe & Moderately severe & 50 & 20 & No response to ice \\
\hline 3 & Very severe & Very severe & 0 & 0 & Not done (uncooperative) \\
\hline 4 & Moderately severe & Moderately severe & 95 & 95 & Not done (perforation) \\
\hline 5 & Moderately severe & Moderately severe & 90 & 90 & Normal \\
\hline 6 & Very severe & Severe & Nil & 45 & Normal \\
\hline 7 & Severe & Very severe & 90 & 45 & Normal \\
\hline 8 & Moderately severe & Moderately severe & 30 & 40 & Normal \\
\hline
\end{tabular}

membrane. Tone decay was absent in all except patient III.52 who was profoundly deaf. Recruitment of hearing was demonstrated in three persons, one of whom had a type II Bekesy audiogram, all others having a type $I$.

Spontaneous or positional induced nystagmus was not identified in any patient and the pendulum tracking test was normal in all. Only one (patient 2) had an abnormal caloric test in which even with ice cold water no responses were elicited.

Two patterns of symptomatology were evident with ototoxic features presenting within one week of the start of treatment in patients 1 and 5 , and only after 1 to 5 months in the remainder. No subject complained of vertigo, but five persons experienced tinnitus with simultaneous onset of deafness.

An adolescent girl (IV.1), who was ostensibly deaf and who had received only prophylactic tuberculous therapy with isoniazid $300 \mathrm{mg}$ daily, was found to be malingering and to have normal hearing. She had previously convinced another institution of her hearing loss and had been issued with a hearing aid which she never used.

\section{Discussion}

The ototoxic complications of all the aminoglycoside antibiotics are well known and similar. The mechanism of ototoxicity at a molecular level is unclear but Spoendlin, ${ }^{4}$ in electron microscopic studies of the inner ears of cats, showed that the aminoglycoside antibiotics may act in a manner akin to their antimicrobial action by inhibiting protein synthesis. In animal investigations, Kehonen ${ }^{5}$ and Engstrom and Kehonen ${ }^{6}$ recognised primary damage by streptomycin to the organ of Corti hair cells followed secondarily by the involvement of the nerve fibres and ganglion cells. This process, which is dose related, has been confirmed in human studies. ${ }^{7}$

The type of deafness in our patients is perceptive and is localised to the end organ (cochlea) as confirmed by audiometry. Also demonstrated were the characteristic initial effects of aminoglycoside toxicity, namely high frequency hearing loss and relatively poor speech discrimination scores. Interestingly, only one of our patients (III.47, patient 2) had abnormal caloric test responses, despite the evidence that the vestibular apparatus is affected before the cochlea in streptomycin sulphate ototoxicity. ${ }^{8}$ However, abnormal vestibular test (ENG) findings have been shown to revert to normal in a significant proportion of patients with vestibular damage of this type. ${ }^{9}$

Although a few reports of deafness attributed to the use of isoniazid, rifampicin, and ethionamide have been reported, the drug most likely to have caused loss of hearing in our patients was streptomycin sulphate. All eight persons received the drug in the dosages recommended for their age and physical status and none had evidence of renal impairment. These deaf subjects all claimed to have had normal hearing before the start of tuberculos is therapy, and this fact was confirmed by unaffected family members, medical officers, and clinic nurses.

Although rare, familial aggregation of susceptibility to streptomycin toxicity has been reported by Prazic et al, ${ }^{1}$ Podvinec and Stefanovic, ${ }^{2}$ and Johnsonbaugh et al. ${ }^{3}$ A genetic basis is suggested by the observation of Donald and Sellars ${ }^{10}$ who encountered a mother and child pair in a study of streptomycin ototoxicity in the unborn child.

As shown in the pedigree (figure), eight persons of both sexes had developed deafness while on antituberculous treatment and three consecutive generations are involved. Only one member (III.50) had similar therapy without deafness ensuing, although four (III.41, IV.1, IV.2, IV.4) received other forms of antituberculous therapy without ototoxicity occurring. Streptomycin therapy had been avoided in these persons when it was recognised by community medical officers that some persons in this family had experienced ototoxic side effects from this drug. The distribution of deaf persons versus those of normal hearing in the treatment group, the male to female ratios, and the apparent generation to generation transmission are consistent with autosomal dominant inheritance of susceptibility to 
cochlear damage. Several members of the family with normal hearing have never been exposed to streptomycin and some of these persons may well possess the faulty gene. They cannot, however, be identified and formal pedigree analysis is impossible. Streptomycin ototoxicity is apparently behaving as an autosomal dominant trait in this family, although this cannot be fully proven with the available data.

Although familial sensitivity to streptomycin seems to be well established, it is very rare, as evidenced by the paucity of case reports. This contention is supported by the experience of community physicians who are involved with the treatment of tuberculosis in the local population. Although they are aware of familial propensity, they regard it as a highly unusual event, and it seems that the condition is genuinely rare.

The hearing acuity of affected members of this kindred is unlikely to improve. However, direct benefits have accrued from the investigation, in that each deaf person has been fitted with an appropriate hearing aid and carefully instructed on its use. Indirectly, future generations of this family will benefit in a preventative manner by the avoidance of all aminoglycoside antibiotics. The appropriate antituberculosis authorities have already been notified of our findings and recommendations.

It is possible to speculate that the wide range of individual variability of ototoxic reactions to streptomycin therapy may be related to inherited characteristics of receptors on the cell membrane of the hair cells within the inner ear. There are many possibilities, including variation in the numbers of receptors or the amount and specificity of enzymes at the receptor site. For these reasons, it could be postulated that familial streptomycin sensitivity is directly related to the membrane characteristics which are under genetic control.

We are grateful to T Heckroodt SRN and Lesley Merckel SRN for organising the family studies and gathering the data on which this report is based. The staff of the Otorhinolaryngology Ward and Audiology Unit of Groote Schuur Hospital, Cape Town, are thanked for their helpfulness and efficiency in coping with a sudden large influx of patients. We thank Mrs June Chambers for typing the manuscript and Greta Beighton for preparing the pedigree. Dr P Dorrington initially clinically assessed and investigated the proband (II.6) and we acknowledge his contribution. This study was supported by grants from the Mauerberger Foundation, the South African Medical Research Council, and the University of Cape Town Staff Research Fund.

\section{References}

1 Prazic M, Salaj B, Subotic R. Familial sensitivity to streptomycin. J Laryngol 1964;78:1037-43.

2 Podvinec PS, Stefanovic P. Surdite par la streptomycine 을 et predisposition familiale. J Fr Otorhinolaryngol 1966; 15:61-7.

3 Johnsonbaugh RE, Drexler HG, Light IJ, Sutherland JM. Familial occurrence of drug-induced hearing loss. Am J Dis Child 1974;127:245-7.

4 Spoendlin H. Streptomycin induced changes in the inner ears of cats. Pract Otorhinolaryngol 1966;28:305.

5 Kehonen A. Effect of some ototoxic drugs upon the pattern and innovation of the cochlear sensory cells in the guinea pig. Acta Otolaryngol [Suppl] (Stockh) 1965;59:208.

6 Engstrom H, Kehonen A. Cochlear damage from ototoxic antibiotics. Acta Otolaryngol (Stockh) 1965;59 171-8.

7 Ballantyne J. Iatrogenic deafness. J Laryngol 1970;84 967-1000.

8 McGee TM, Olszewski J. Streptomycin sulphate and dehydrostreptomycin toxicity. Arch Otolaryngol 1982;75: $25-41$.

9 Fee WE. Aminoglycoside ototoxicity in the human. Laryngoscope 1980;90:(suppl).

10 Donald PR, Sellars SL. Streptomycin ototoxicity in the unborn child. $S$ Afr Med J $1981 ; 60: 316-8$.

Correspondence and requests for reprints to Professor $\mathbf{P}$ Beighton, Department of Human Genetics, Medical School, University of Cape Town, Observatory 7925, Cape Town, South Africa. 